\title{
Transferability of Industrial Management Concepts to Healthcare Networks
}

\author{
Dario Antonelli ${ }^{1}$, Agostino Villa ${ }^{1}$, Bart MacCarthy ${ }^{2}$, and D. Bellomo ${ }^{3}$ \\ ${ }^{1}$ Department of Production Systems \& Economics, Politecnico di Torino, Italy \\ agostino.villa@polito.it, dario.antonelli@polito.it \\ ${ }^{2}$ Operations Management Division, Nottingham University Business School, UK \\ bart.maccarthy@nottingham.ac.uk \\ ${ }^{3}$ Azienda Sanitaria Locale ASL-AT, Asti, Italy \\ bellomo@asl.at.it
}

\begin{abstract}
The paper presents the preliminary results of a RTD project devoted to the transfer of concepts and methods, originally developed in the industrial area, to the subject of healthcare services where a profitable application is envisaged. The approach is based on drawing out the analogies between supply chains, composed by a network of resources connected by transport devices, and the local networks of healthcare services (family doctors, specialists, ambulatories, first aid centers, hospitals) to which patients are directed. The crucial task of efficiently and effectively managing a territorial network of healthcare service centers is modeled by using control theory concepts, and application conditions are discussed.
\end{abstract}

\section{Introduction}

Governments of developed countries are presently facing severe budget problems: among them, one of the most critical is the management of the national and regional healthcare systems, to assure a fair service level to the population under reasonable costs. Unless relevant adjustments will be applied, incidence of the healthcare costs on the GNPs will become explosive in a few years, thus forcing a reduction of the public covering of expenses.

With such a scenario, recent studies ([1] and [2]) expressed the necessity of a deep review not only of how to finance the healthcare costs, but mainly of how to plan the service capacity through suitable funding installments and to control the quality level of provided services. Then new tools and procedures for the management of healthcare services provided by local Healthcare Territorial Agencies (HTA) appear to be mandatory. Their utilization could allow a real management of the crucial nodes of the healthcare systems of any country because the reduction of costs does not be obtained at the population's expense. Till now management "protocols" able firstly to 
estimate, from the large data base of a HTA, the values of some Key Performance Indicators (KPIs) which could evaluate the service quality, efficiency and cost, secondly, to suggest criteria and procedures for the planning and control of the HTA operations, have not yet been established. To be more explicit, performance evaluation of individual services, like Emergency Rescue, Hospital Divisions, has been already applied but there is a lack of results referring to the performance evaluation of the whole health network. In the industrial sector, since many years, the attention has been focused on the performance of the entire Supply Chain more than to the local optimization of the individual processes.

This paper will utilize the experience acquired by the partners of the CODESNET ${ }^{1}$ project in the field of the performance evaluation of SME networks, to the aim of analyzing the transferability of industrial management methods to the service planning and control in the healthcare sector. The scope is to investigate the possibility of transferring the industrial methods of network performance analysis to a different kind of network, a HTA made of a number of local healthcare service providers of different types, namely: hospitals, pharmacies, medical centres, panel doctors, diagnosis laboratories, etc. The main idea is the application of concepts of supply chain management, such as localization and networking, to a HTA. Here, localization means to delegate the administration of healthcare centres to administrative units on a local scale, provided that a common planning of expected service capacity and level has been centrally defined; networking refers to a comprehensive management of all the health related services in order to exploit the advantages of collaboration and information sharing.

New models for the management of HTA could apply the principles of optimal planning of the network capacity and of real-time (in practice, day-by-day) control through feedback by measuring appropriate KPIs. Dealing with the transferability of the mentioned industrial planning \& control concepts and methods to healthcare sector, it must be noted that direct transfers are not always successful: systems, concepts and techniques often need specific interpretation, adaptation and adjustment for the different application domain. Special care must be used if the "subject" of the service is a person, having proper decisional autonomy, as in the case of a patient, even if such a decisional autonomy is often limited by a reduced knowledge of the service system itself. In this case there is the risk of a dynamic adaptation of the system measures to the chosen KPIs in order to increase the attractiveness of the service.

The paper begins with the description of the respective features of industrial supply chains on one side and healthcare service networks on the other. There is a "theoretical part" that outlines the main ideas of the transferability of management methods from industrial sector to the healthcare system by highlighting the analogies and the differences between the two networks. Eventually there is an "operative part", in order to outline main contents and potential usefulness of a HTA Management Protocol for supporting the HTA manager and the local service controllers.

${ }^{1}$ CODESNET - COllaborative DEmand \& Supply NETworks is a Coordination Action funded by the European Commission in the 7th FP, chaired by A. Villa (re: www.codesnet.polito.it). 


\section{Specific Characteristics of a Supply Chain}

Health networks can be analyzed by similarity with the other industrial service networks. The most common industry network is the supply chain. For industrial supply chain it is intended a network of production and distribution services connected through agreements, which performs the functions of procurement of materials, transformation of them into intermediate and finished products, and finally distribution of final products to customers [3].

Supply chain management has been viewed as the decision-making structure to be developed to make possible a real interaction among firms operating concurrently. In terms of a conceptual model, a supply chain can be graphically represented such as a graph of production stages and markets, alternatively connected: it means that each node "production stage" can be only connected with nodes "market" both upstream and downstream [4] and [5]. Owing to its multi-stage structure, supply chain management is referred to as a typical large-scale management problem since each component firm can be viewed as an "agent", whose management decision should be planned through a negotiation with other agents, namely with its own suppliers (upstream) and buyers (downstream). Then the organization of a supply chain must solve the following two complementary problems [6]: (a) define efficient and effective negotiation procedures for each "market"; (b) define a common coordination strategy for all production stages, such to assign to each stage a reference plan assuring a global efficiency/effectiveness of the supply chain.

These two management problems must be solved in a coordinated way, such as to avoid conflicts between the coordination action (to be centrally planned) and the local negotiations among consecutive production/service stages.

\section{Specific Characteristics of a Healthcare System}

In a territorial healthcare system - as the HTA - three different "agents" are present:

1. the HTA manager, with the scope of planning the service capacity of the healthcare service centres in a specific local region;

2. the healthcare service providers, each one being committed to provide a given healthcare service within a HTA centre;

3. the set of end-users - the patients - each one asking for a given healthcare service, either autonomously or driven by a provider.

The following types of services are considered:

1. "hospital service", summarizing in this concept all different functions which could only be provided there upon request of a patient;

2. "intermediate service", such as local consulting, health status testing;

3. "input services", as family doctor and specialist.

The most important service structures composing the healthcare system can be represented as components of a network as the one sketched in Fig. 1. 


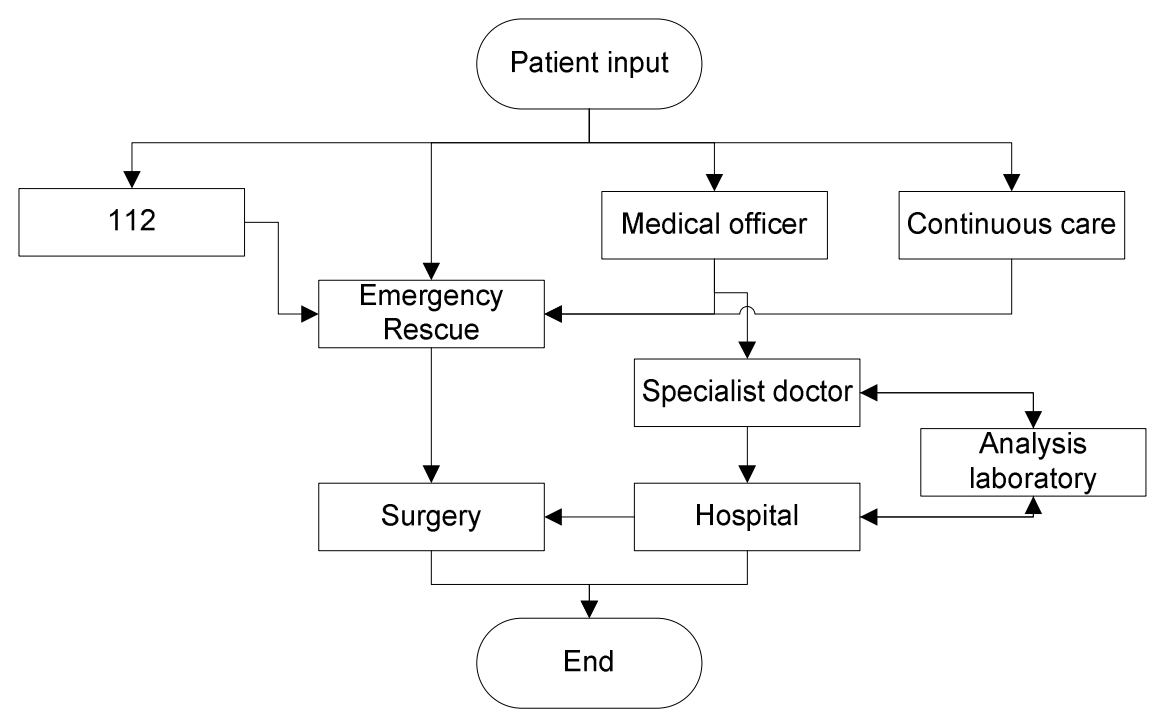

Fig. 1. A generalized network of healthcare services

The hospital plays the role of the main service (here considered in an aggregated form), which collects flows of patients coming from several upstream service, namely: day-hospital, local consulting services, considered as intermediate services of the network; family doctors (medical officers) and specialists, usually acting as the upstream services or input services for patients. Upstream services have to address their patients to the downstream ones, according to the service capacity values of these latter.

Differently from industrial supply chain, in a HTA it is important to take into account the geographical localization of service providers as the patients are spread on the territory. As an example, in Fig. 2, the distribution of accesses to the Asti hospital, in the northern Italy, shows that the attractiveness of the service 'hospital' is a complex combination of the quality of the service offered, the availability of efficient communication infrastructures and of the distance from the patient.

From a theoretical point of view, Fig. 1 gives an illustration of how services could be used in various sequences by patients, in industrial terminology the "routing". It is assumed that "precedence conditions" exist among services, that means conditions which make necessary to ask for specific sequences of services often denoted "healthcare protocols" (that is the medical equivalent of the routing). The set of healthcare protocols at disposal in a HTA defines the graph of Fig. 1, where nodes are the services and each link represents the precedence conditions between two services. The flows of patients asking for that sequence of services are assigned by either monitoring or estimating the number of patients per unit time accessing both services, according to all "protocols" including the two services themselves. 


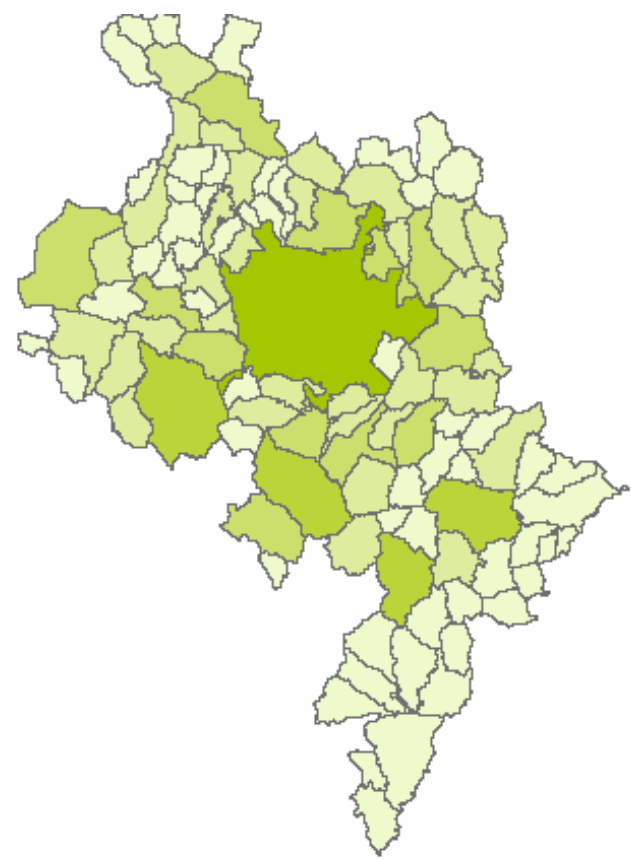

Fig. 2. Maps of attractiveness of the Asti hospital (darker area mean more accesses)

\section{Analogies for Transferability}

The scheme of healthcare service network of Fig. 1 and 2 presents several analogies with a supply chain model describing production operations. The following Table 1 gives evidence of these similarities between healthcare system functionality and production network operations.

The aim of this table is to illustrate correspondences both with regard to the "agents" acting inside the two systems, and their interactions, as well as their respective goals. The scope is to envisage the similarities but also to recognize the differences: these last ones will give the right suggestions for a correct transferability of models and tools from the two sectors.

By Table 1, indeed, evident similarities can be envisaged, but also a significant difference. The real goal of a patient is to find a service which could assure that a desired health status could be reached. This target can be reached by the cooperative actions of both the patient - who must be able to explain symptoms and ready to carefully follow prescriptions - and the service provider - who must be efficient in using right competence to make diagnosis. Cooperation, instead of the competition governing industrial interactions, and the territorial distribution of one type of agents (the potential patients) are the two milestones of healthcare systems: they call for a better specification of this sector itself. 
Table 1. Similarities/differences between industrial and healthcare systems

\begin{tabular}{|c|c|c|}
\hline Items & Industrial Network (SC) & HTA \\
\hline \multirow[t]{2}{*}{ Agents } & (a) Supplier & (a) Service provider \\
\hline & (b) Client & (b) Patient \\
\hline $\begin{array}{l}\text { Agents' position in } \\
\text { the process }\end{array}$ & $\begin{array}{l}\text { concentrated-parameter } \\
\text { process: agents are } \\
\text { associated to nodes of the } \\
\text { network. }\end{array}$ & $\begin{array}{l}\text { mixed-parameter process: } \\
\text { service providers are } \\
\text { associated to HTA, } \\
\text { patients are distributed on } \\
\text { the territory. }\end{array}$ \\
\hline Agents interactions & $\begin{array}{l}\text { Negotiation to manage } \\
\text { competition between the } \\
\text { two types of agents }\end{array}$ & $\begin{array}{l}\text { Cooperation to assure the } \\
\text { most effective service. }\end{array}$ \\
\hline Agents decisions & $\begin{array}{l}\text { Each type of agent } \\
\text { chooses the counterpart } \\
\text { based on convenience }\end{array}$ & $\begin{array}{l}\text { The patient chooses the } \\
\text { service provider who } \\
\text { seems to be more credible }\end{array}$ \\
\hline Agents goals & $\begin{array}{l}\text { To maximize agents' own } \\
\text { profits. }\end{array}$ & $\begin{array}{l}\text { The patient wants to reach } \\
\text { a desired health status; the } \\
\text { provider aims to assure a } \\
\text { desired performance } \\
\text { minimizing service costs. }\end{array}$ \\
\hline
\end{tabular}

\section{Modeling Healthcare System by Use of Industrial Concepts}

The similarities and the differences outlined in Table 1 allow to develop a model of the healthcare system - specifically referred to a HTA - by using industrial concepts. The model is generated by making reference to the classic functional diagram of a SC.

The model of the healthcare service network does not describe the HTA as a network of resources; then, it does not account for the management of the resources (that are the service centres) neither for the choice of specific resources made by patients.

To this extent, it is necessary to translate the main functionalities of the three agents into proper management decisions, namely:

i. planning decisions adopted by the HTA network manager;

ii. decisions to assure the quality level of services and the service centre attractiveness, made by the service providers;

iii. decisions to choose the most credible provider and the most accessible service centre, done by the patients.

By using this classification of decision types, a model of the HTA management can be stated in terms of a block diagram (and related planning/control actions) directly derived from the industrial application of control theory - as represented in Fig. 3.

This figure gives a clear view of the application of concepts developed in the frame of Control Theory and frequently applied in industrial management, to the healthcare sector. The application indeed describes the interactions among the three agents of a HTA in terms of respective decisions concerning either the planning and local control of the offered services, or the choice of some service centres, and the service providers there operating. 


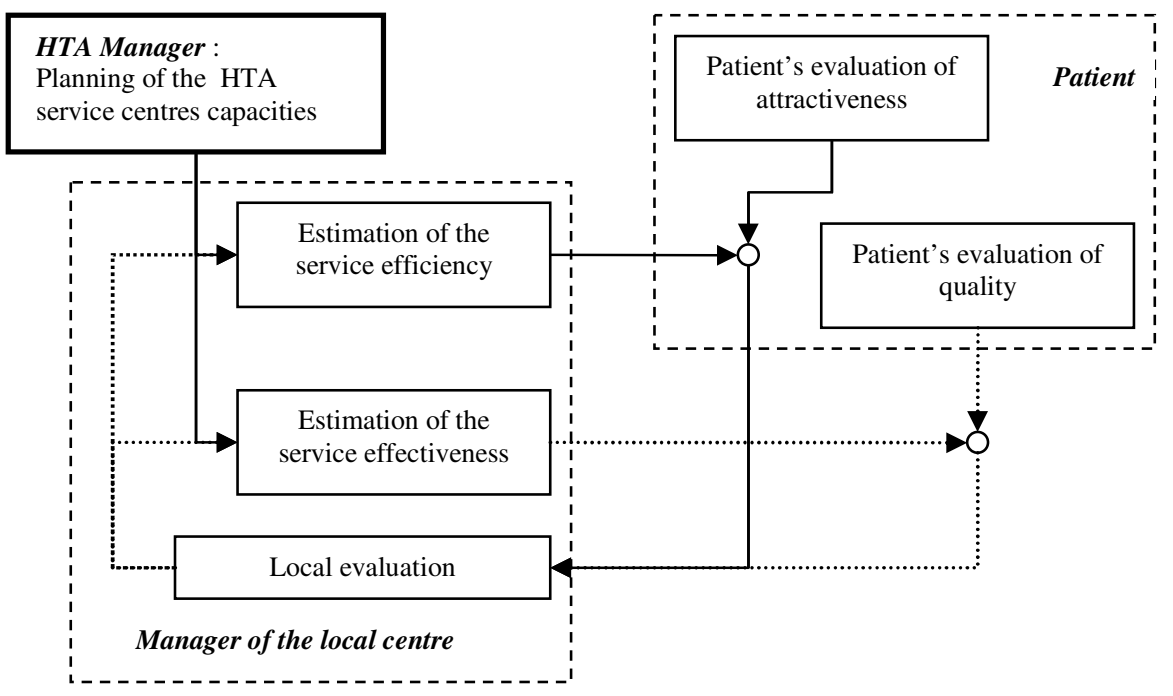

Fig. 3. A control diagram of the interactions among the three HTA agents

According to the above block diagram, the HTA manager has the task of programming the service capacities of the HTA centers, thus assuring a planned attractiveness and a sufficient quality level. To this aim, the HTA manager can use information concerning the population distribution in the HTA territory, and evaluate the expected flows of persons towards the centers (e.g. by gravity models and methods to subdivide a region into sections depending on the transport connections, as in Iwainsky et al, 1986).

Considering a local service center, the center manager has to transform the centralized plans into time-by-time controls. On one hand, it has to assure that the attractiveness of its own center be as high as possible, through a careful management of the incoming patients' flows (e.g., through scheduling of arrivals and services, by applying industrial scheduling tools as presented in [9]). On the other, it has to monitor the offered quality (e.g., by Quality Control tools, as in [10]). Both actions should be designed - and applied - by tacking into account that the local manager's decision is a typical feedback control, driven by information both on the currently offered service types and levels, and on the number of patients who decided to use this same service center.

The utilization of a service center by a patient depends on two main considerations (as in case of a "client" of an industrial service): first, the possibility of a fast utilization of the service; then, the a-priori evaluation of the service quality, usually based on the "credibility" of the provider and the information previously received on the efficiency of the center personnel. The industrial approach is based on the estimation of the patients flows over the network of service centers by formally modeling the preferences of patients (e.g., as in choosing among alternative products by a AHP procedure, as shown in [11]) and their dynamics over the service network (e.g., by queue theory models, as in [12]). 


\section{Conclusions}

The presented block diagram of Fig. 3 gives a scheme of management for a territorial healthcare system concurrently based both on the goals of the central manager, efficiency and service capacity, and on the needs of the patients, service quality and effectiveness of the cure.

The real application of this scheme requires two main actions: first, to define central strategies easy to be understood also locally; second, to support local managers in their crucial task of understanding the patients desires and choices. The application of techniques successfully experienced in the industrial sector seems to be promising and has originated the RTD projects currently developed at Politecnico di Torino, funded by the Italian Ministry of University and Research (PRIN). The research is also supported by the Compagnia San Paolo of Torino - Italy.

\section{References}

1. Starfield, B., Leiyu, S.: Policy relevant determinants of health: an international perspective. Health Policy 60, 201-218 (2002)

2. Organizational Environment and Content Requirements for Health Promotion and Care Information, http: / / www. virtual . epm. br/material/heal thcare/A03 .pdf

3. Bergman, E.M., Feser, J.E.: Industrial and Regional Clusters: Concepts and Comparative Applications (1999), http: / / www.rri.wvu.edu/WebBook/BergmanFeser/

4. Akif, J.C., Blanc, S., Ducq, Y.: Comparison of methods and frameworks to evaluate the performance of supply chains. In: 4th IFIP Int. Workshop on Performance Measurement, Bordeaux, June 27-28 (2005)

5. Lin, C., Chow, W.S., Madu, C.N., Kuei, C.H., Yu, P.P.: A structural equation model of supply chain quality management and organizational performance. Int. J. of Production Economics 96, 355-365 (2005)

6. Villa, A., Antonelli, D.: A Road Map to the Development of European SME Networks Towards Collaborative Innovation. Springer, London (2008)

7. Healthcare Informatics Alliance, http: / /www2.wmin.ac.uk/hscmg/pdf/ WestFocus_Talk_31-05-2006_Millard.pdf

8. AHRQ - Agency for Healthcare Research and Quality, http: / / www.qualityindicators.ahrq.gov/

9. Brandimarte, P., Villa, A.: Advanced Models for Manufacturing Systems Management. CRC Press, Boca Raton (1995)

10. MacCarthy, B.L., Wasusri, T.: A review of non-standard applications of statistical process control (SPC) charts. The International Journal of Quality \& Reliability Management 19(3), 295-320 (2002)

11. Dyer, R.F., Forman, E.H.: An Analytic Approach to Marketing Decisions. Prentice Hall, Englewood Cliffs (1991)

12. Papadopoulos, H.T., Heavey, C., Browne, J.: Queueing Theory in Manufacturing Systems Analysis and Design. Chapman \& Hall, London (1993) 